\title{
Experiencias literarias que propician una sinergia emotiva entre padres y alumnos
}

\author{
(Literary Experiences that Foster an Emotional Synergy between \\ Parents and Students)
}

MARÍA DOLORES FLORES MONROY

Colegio de Bachilleres delo Estado de Sonora, Sonora, México

\section{Volumen 1, Número 1}

Edición especial. Febrero de 2016

p. $235-244$

Este número se publicó el 5 de febrero de 2016

Artículo recibido: 25 de septiembre de 2014

Artículo aprobado: 30 de noviembre de 2015

ISSN: 2448-5942, doi: https://doi.org/10.36799/el.v1i1.34

Citar este artículo como:

Flores Monroy, M. D. (2016). Experiencias literarias que propician una sinergia emotiva entre padres y

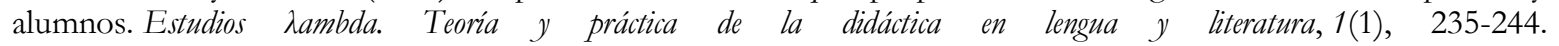
https://doi.org/10.36799/el.v1i1.34

Derechos de autor: El autor o autores conservan en todo momento sus derechos morales y patrimoniales sobre la obra; la obra no se puede alterar, transformar o ampliar; siempre debe reconocerse la autoría del documento

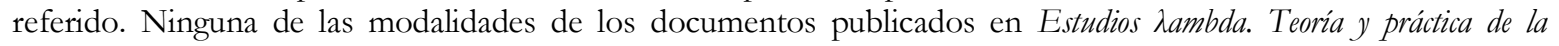
didáctica en lengua y literatura tienen fines comerciales de naturaleza alguna.

Los contenidos de este artículo están bajo la licencia de Creative Commons Atribución No Comercial- Sin Derivadas 4.0 Internacional @() 


\title{
Experiencias literarias que propician una sinergia emotiva entre padres y alumnos
}

\author{
(Literary Experiences that Foster an Emotional Synergy between \\ Parents and Students)
}

MARÍA DOLORES FLORES MONROY ${ }^{1}$

\begin{abstract}
RESUMEN
El presente artículo expone una experiencia didáctica consistente en la ideación de un diseño de aprendizaje aplicado a cincuenta estudiantes preparatorianos, con la involucración de los padres y con una duración de un semestre. Tal experiencia consistió en la redacción de una narración sencilla y breve. La metodología cualitativo-interpretativa consistió en la redacción de una anécdota que posteriormente sirvió de base para redactar una fábula, teniendo como co-autores a papá y mamá. Los resultados esperanzadores y optimistas se reducen a demostrar que el alumno de bachillerato puede mejorar su redacción si se incluye como apoyo de aprendizaje el apoyo de sus padres.
\end{abstract}

PALABRAS CLAVE: Experiencia didáctica, participación de padres, trabajo compartido, afecto, emoción con sinergia.

\begin{abstract}
The present paper expounds a didactic experience in the devising of a learning design applied to fifty high school students with parents' involvement during a whole semester. Such experience consisted in asking the students to make up the composition of a simple and short narrative. Methodology, qualitative and interpretative, was about the writing of an anecdote which was lately considered as the base for transforming it later as a fable, having as co-writers mom and dad. The results, optimistic and hopeful, show that the adolescent high school student can improve his writing when his/her parents' help is included as support.
\end{abstract}

KEYWORDS: Didactic experience, parents’ participation, team work, affection, emotion with synergy.

\footnotetext{
${ }^{1}$ Lic. en educación media en el área de español. Docente en el colegio de Bachilleres delo Estado de Sonora. mariadolores96@yahoo.com.mx
}

Artículo recibido: 25 de septiembre de 2014

Artículo enviado a corrección: 12 de octubre de 2015

Aprobado: 30 de noviembre de 2015

Flores doi: https://doi.org/10.36799/el.v1i1.34Volumen 1, Número 1, Año 2016, ISSN: 2448-5942 


\section{INTRODUCCIÓN}

A lo largo de mis años de docente me he enfrentado a una problemática variada tanto en mi enseñanza como en el aprendizaje de mis alumnos. Últimamente he visto agravarse un aspecto que requiere atención inmediata. Aunque la he socializado con mis colegas no había encontrado aún una estrategia convincente y efectiva para enfrentarla. Ahora me parece que he logrado realizar algo novedoso, lo cual expongo a su consideración. Hace dos o tres años realicé un ejercicio de redacción de un relato con cincuenta alumnos en un solo grupo con la finalidad de desarrollar en ellos las capacidades comunicativas a través de la producción de textos. A continuación presento en sucintos apartados los principales elementos que participaron en mi experiencia didáctica.

El Plantel Villa de Seris siempre se ha caracterizado por destacar en actividades académicas culturales y deportivas. Tanto los Maestros como los alumnos nos enorgullecemos de pertenecer a esta institución educativa por su alto valor y su sentido de pertenencia; sin embargo, actualmente se ha observado en sus estudiantes un desánimo en cuanto a las actividades académicas respecto a las materias de Lenguaje y comunicación, mismas que se proyectan a través de los resultados obtenidos en las evaluaciones recientes. Aunque seguimos obteniendo buenas calificaciones en pruebas externas, al interior del Plantel hemos observado algunas deficiencias en las nuevas generaciones que nos están llegando.

Ya no contamos con el clásico alumno que llegaba a la escuela a aprender, que entraba antes que el maestro y permanecía atento a sus indicaciones, ahora batallamos con alumnos al grado tal que casi los tenemos que obligar a entrar en el aula y sobre todo a permanecer en ella.

\section{JUSTIFICACIÓN}

Son muchas las problemáticas detectadas en nuestros jóvenes estudiantes, pero sólo pretendemos atacar aquellas que son de nuestra competencia. Enterados de otras experiencias similares en las que se involucró a los padres en el aprendizaje de sus hijos en escuela primaria, y que produjeron resultados sobresalientes en California, por ejemplo (Avendaño, 2004;), y alentados por explorar en nuestra región con una perspectiva parecida y optimista, consideramos importante como medida significativa y heurística, involucrar de manera muy

Flores doi: https://doi.org/10.36799/el.v1i1.34Volumen 1, Número 1, Año 2016, ISSN: 2448-5942 
especial y contundente a los padres de familia en el aprendizaje de nuestra materia de lenguaje y literatura. Nuestro supuesto parte del hecho de que es nuestro quehacer sacar adelante a los jóvenes en aspectos académicos, concretamente en mejorar los hábitos de estudio y la comprensión lectora, pero en este caso, con mayor razón y fundamentación dado que hemos detectado que la mayor parte de los alumnos enfrentan problemas de aprendizaje porque, dentro de otros, tienen problemas familiares, mismos que no les permiten concentrarse y dedicarse $100 \%$ al estudio.

Resulta curioso el hecho de que la mayor parte de la bibliografía consultada sobre el involucramiento de los padres en el aprendizaje de sus hijos, se ubica en la escuela elemental y en la pre-primaria (McLaughlin, 1987; Weiss, Bouffard et alia, 2009). De la escuela secundaria casi no se reportan estudios y de la preparatoria menos. La pregunta obligada entonces es cómo es que hemos recurrido a este factor de intervención de terceros, los padres, pero en el nivel de escuela media superior. En parte ya se ha mencionado en el parágrafo anterior la grave problemática por la que atravesamos, y en consecuencia tenemos como maestros que arriesgarnos a explorar nuevas alternativas, nuevos métodos, nuevas estrategias, y por fin el apoyo de otras personas con las que establezcamos y aprovechemos esa sinergia afectiva entre padres y sus hijos, nuestros alumnos.

Esos problemas familiares van desde lo económico hasta las cuestiones muy íntimas del núcleo familiar como lo son: falta de atención al adolescente por divorcio de los padres, mal trato físico, mal trato psicológico, embarazo prematuro, drogas, entre otros.

Ante estas contrariedades, nos vemos en la necesidad de cambiar de estrategias. Antiguamente nos preocupaba la baja del rendimiento escolar; cuando lo veíamos hablábamos que los promedios de 100 disminuían a 90, pero en la actualidad es mucho más preocupante ver el desinterés mostrado por los alumnos, reflejado en el alto índice de reprobación que se está presentando en las materias del área de lenguaje, y que ha llegado casi al $40 \%$ de nuestros alumnos.

Todos sabemos que en el proceso educativo como en la sociedad, todo está en constante cambio, formamos parte de la sociedad posmodernista (Lipovetsky 1998, Bauman 1984). Este período va marcado por la pérdida de muchas características que también vemos

Flores doi: https://doi.org/10.36799/el.v1i1.34Volumen 1, Número 1, Año 2016, ISSN: 2448-5942 
en nuestros jóvenes: la pérdida de valores idealistas y humanísticos, la falta de solidaridad, la aspiración a la justicia, la defensa del oprimido, la cooperación en causas nobles y tradicionales. En lo que a nosotros como docentes concierne, el trabajar con adolescentes implica una gran responsabilidad, pues son muchos los factores que intervienen en dicho proceso y que deben considerarse para planear, diseñar, estructurar, adecuar e implementarse en el sistema educativo, por lo tanto, la docencia implica cosas muy distintas a las de hace algunas décadas. Hoy, se visualizan nuevos escenarios globalizados y fríos a los que el docente debe enfrentarse para formar, con calidad y calidez a esas generaciones competitivas y digitalizadas de la actualidad.

La problemática que últimamente se ha vuelto casi clásica en el área de Lenguaje en el Colegio de Bachilleres del Estado de Sonora Plantel Villa de Seris, es el hecho de que a los estudiantes no les gusta leer ni escribir, y ven las materias correspondientes a esta área, como materias ancilares, secundarias, "de relleno".

Sin el ánimo de restarle a las nuevas tecnologías de la información, el mérito y el reconocimiento alcanzado, creo que en el caso que nos ocupa como docentes en el nivel medio superior, es muy difícil compaginar la escritura propiamente dicha, con la escritura que practican nuestros alumnos en las redes sociales.

Lo anterior no es precisamente la razón de ser de este trabajo; sin embargo, la tecnología, por sí sola, se ha vuelto un problema para las materias de lenguaje, y por lo tanto, pareciera implicar un reflejo en la degradación de estas materias. Es por ello que el docente debe buscar nuevas formas de trabajar. Por ejemplo, consideramos que la materia de literatura, para mi gusto y perspectiva, es la materia que en un grado mayor sensibiliza a nuestros digitalizados estudiantes, al volverlos más humanos. Pero la tarea que señalo no corresponde a una sola persona, puesto que para mejorar la calidad educativa se requiere de la colaboración de todos aquéllos que estamos interesados en lograrla, en este caso, me refiero principalmente al maestro y a los padres de familia, quienes, finalmente, somos los más cercanos al alumno en este proceso.

Flores doi: https://doi.org/10.36799/el.v1i1.34Volumen 1, Número 1, Año 2016, ISSN: 2448-5942 


\section{METODOLOGÍA (ACTORES, OBJETIVO, METAS, PROPÓSITO, MEDIOS)}

Paso ahora a exponer el caso particular en la que me vi directamente involucrada. El trabajo de investigación que expongo se inscribe en una metodología cualitativo- interpretativa. En una primera fase se llevó a cabo la redacción de una narración sencilla y breve; es decir, se solicitó al alumno que redactara una anécdota, un suceso que hubiera experimentado. Cabe aclarar que en segundo semestre de preparatoria los alumnos aprenden a redactar anécdotas, por ello no fue necesario retomar el tema para explicarles la meta solicitada y la justificación correspondiente.

Cuando empecé a trabajar esta actividad, solamente tenía la intención de ayudar a mis alumnos en la comprensión lectora de los textos de mi clase, y en producir textos literarios narrativos, específicamente, fábulas.

Posteriormente, cuando observé que la mayoría se quejaba en sus escritos por la falta de interés que mostraban los padres hacia el trabajo realizado por sus hijos, me pareció interesante involucrar a los mismos padres en el trabajo de sus hijos-estudiantes con la finalidad de que conocieran las dificultades que representa el ser estudiante en la actualidad. Solicité por comunicados escritos a los padres mi intención para con sus hijos. Enseguida convoqué a una reunión de padres en el plantel donde les expuse el procedimiento. La mayoría no tuvo problemas pero algunos de los padres estaban indecisos por desconocimiento; no se atrevían directamente a comprometerse y me preguntaban que si yo les podía ayudar. Me ofrecí a ayudarles en lo que pudiera y también les dije que estaba a su disposición para que me visitaran cuando lo creyeran conveniente. Así pues constaté que aunque los padres no tuvieran una formación escolar considerable, sí podían motivar a sus hijos en la realización de la tarea, aunada a una previa explicación de escrita sobre la tarea, de mi parte.

Debido a la temática que manejaron los jóvenes, en una segunda fase trabajé más específicamente con la redacción de una anécdota (un cuento de la vida real, les trataba de explicar) en la que hubieran sentido la necesidad de platicarla con alguno de sus amigos, padres o hermanos. En esta ruta metodológica, se solicitó que formaran equipo con mamá y papá para reescribir la historia de la anécdota pero ahora utilizando un diferente género narrativo, la estructura de la fábula. Todos los trabajos debían entregarse firmados por todos los integrantes

Flores doi: https://doi.org/10.36799/el.v1i1.34Volumen 1, Número 1, Año 2016, ISSN: 2448-5942 
del equipo. Se solicitó que inventaran un pseudónimo con el cual firmarían sus trabajos, el cual estaría registrado en la lista de asistencia para que yo, como docente y guía, pudiera identificarlos. En clase dimos lectura a los trabajos presentados.

\section{Actores}

En esta actividad participamos tres actores: alumnos, padres de familia y una servidora, la profesora guía de la actividad.

- 50 Alumnos de tercer semestre. Grupo 301 V, Ciclo escolar 2012-2013, Colegio de Bachilleres del Estado de Sonora, Plantel Villa de Seris, materia de Literatura 1.

- 35 Padres de familia de los alumnos correspondientes al grupo antes mencionado.

- 1 Docente, María Dolores Flores Monroy.

\section{Objetivo}

Desarrollar en el estudiante habilidades comunicativas por escrito con base en el uso de competencias lingüísticas para interpretar, crear y valorar textos literarios, así como para enfrentar diversas situaciones cotidianas, académicas y familiares de manera afectiva en su contexto situacional mediante la expresión literaria.

\section{Metas}

La producción de textos literarios individuales, específicamente la redacción de fábulas a través de pasos: primero la identificación de las características de la misma, y luego los personajes, la estructura, el conflicto y la conclusión. Terminamos con una fábula completa incluida la moraleja.

\section{Propósito}

Motivar a los a los alumnos mediante diversas técnicas pero con una estrategia más, el involucramiento de los padres de familia para que le encontraran sentido a escribir textos creativos, destacando la importancia de la imaginación y la fantasía, es decir, de la literatura en todos los ámbitos de nuestra vida.

\section{Medios}

- Un texto como la anécdota.

- Un texto como la fábula.

Flores doi: https://doi.org/10.36799/el.v1i1.34Volumen 1, Número 1, Año 2016, ISSN: 2448-5942 
- Módulo de Literatura 1, manual de enseñanza en el Colegio de Bachilleres

\section{RESULTADOS}

Algunos de los resultados obtenidos: en primer lugar, se logró que identificaran las semejanzas y diferencias estructurales entre la fábula y la anécdota. Se realizó una autoevaluación a través de una lista de cotejo.

De 50 alumnos, 35 aceptaron, es decir, se comprometieron con la actividad y de esos 35, 28 trabajaron correctamente, como se había acordado. Los otros 7 escribieron sobre otros subgéneros como capítulos para una novela, otros sobre un diario y algunos más escribieron leyendas o cuentos.

Hubo 15 alumnos que no se sintieron con la confianza de pedir a sus padres que les ayudaran en esta empresa y optaron por trabajar solos en el desarrollo de otro subgénero. Por ejemplo, una alumna escribió tres capítulos de una novela; 13 escribieron un diario y uno más, un cuento que, posteriormente ganó un segundo lugar en un concurso interno.

Como dato adicional, pude observar que a nuestros alumnos les hace falta mucha atención por parte de los padres de familia, tal vez no sea un dato nuevo en el ámbito educativo, pero sí lo es en nuestro plantel, porque, como se dijo al inicio de este escrito, estamos acostumbrados a trabajar con muy buenos alumnos y de los mejores promedios a nivel estatal en materias a las que yo les llamo "frías y calculadoras" como Matemáticas, Física y Química, pero ¿qué pasa con las materias "cálidas” o "con calor humano” como Literatura, Ética y Valores, Taller de Lectura y Redacción, etc.? Ahora sé que muchos de nuestros alumnos trabajan solos y no precisamente porque lo deseen, sino más bien, porque los padres están como nosotros, creyendo que los estudiantes son autosuficientes y no necesitan más apoyo que los libros, el maestro e internet.

Algunos trabajos se publicaron en una página que hicimos especialmente para esta actividad, pero por razones extrañas y que no viene al caso mencionar, no pude conservar. El espacio se llamaba "Letras cambiantes".

Flores doi: https://doi.org/10.36799/el.v1i1.34Volumen 1, Número 1, Año 2016, ISSN: 2448-5942 
Actualmente, cuento con una página de internet a la que cariñosamente llamo "Radio Seris. La voz de los campeones" ya que nuestro plantel es Colegio de Bachilleres, plantel Villa de Seris y es en esta página donde alcancé a publicar uno de los trabajos mencionados, en un espacio al que le llamé "Letras mágicas”.

\section{CONCLUSIONES}

Estamos conscientes que la problemática a la que nos enfrentamos no es exclusiva de México, también la padecen abundantes países de la Unión Europea e incluso en los Estados Unidos de América. Estamos inmersos en una nueva etapa del último capitalismo mejor conocida por los sociólogos como posmodernismo (Lipovetsky, 1986) y también como la etapa líquida (Bauman, 2006). En virtud de la aparente falta de referentes y de valores todo esfuerzo por superar el actual impasse educativo será bien venido.

El objetivo se logró, la mayoría de los estudiantes identificó, practicó y disfrutó de la narración.

Pienso que este trabajo me servirá para realizar una investigación formal, es decir, iniciar con la aplicación de un diagnóstico, un instrumento que me sirva para medir la respuesta de los padres, y el avance de los estudiantes en el desarrollo de la misma. Refrendamos, pues, que el reporte de la experiencia didáctica se centra en una pequeña aproximación exploratoria sobre esta posible y potencial mina para involucrar a terceros. De alguna manera debo matizar los resultados y la metodología que realicé en este trabajo. Nos hemos iniciado en la investigación a pesar del poco tiempo de que disponemos como profesores catedráticos. Una investigación de campo debe - entiendo bien, ahora- formularse con variables, hipótesis, grupos testigos, etcétera. Creo que el presente ha sido un buen comienzo.

Fue muy gratificante ver a tanto joven escribiendo sus ideas que, posteriormente, se mezclaron con las de sus padres; además, la actividad generó cierto interés hacia la lectura, pues todos tenían un trabajo de otro compañero para leer y evaluar.

Flores doi: https://doi.org/10.36799/el.v1i1.34Volumen 1, Número 1, Año 2016, ISSN: 2448-5942 
Al finalizar la actividad, los alumnos mostraban tanto agrado, que me preguntaban cómo hacer para proteger sus escritos, querían registrarlos con derecho de autor.

También es bueno aclarar que de esos 38 alumnos que trabajaron con papá y mamá, no les fue fácil a todos convencer a la familia de hacer tareas escolares de este tipo, pues lo más usual es ayudar a los hijos a resolver problemas matemáticos sin pensar que para ello están los medios como la calculadora o la misma computadora, aunque lo más necesario sea, desde mi punto de vista, ayudar a resolver problemas emocionales donde el mejor medio, si no es que el único, es la familia.

\section{REFERENCIAS BIBLIOGRÁFICAS}

Alcántar Ramírez, Enrique. "La participación de los padres de familia en el proceso escolar de los alumnos de enseñanza media superior”. BCS: Gobierno del Estado de Baja California Sur. Secretaría de Educación Pública. Dirección de profesiones, Educación Media Superior y Superior. Universidad Pedagógica Nacional. Unidad 03, 2009.

Avendaño, Alma. "Educación de padres, camino hacia el mejoramiento académico de los estudiantes de la escuela Lincoln de Escondido California. Tesis no publicada. Departamento Letras y Lingüística, Universidad de Sonora, 2000.

Bauman, Zygmunt. Vida líquida. Barcelona: Paidós Ibérica, 2006.

Lipovetsky,Gilles. La era del vacio. Barcelona: Anagrama, 1986.

McLaughlin, Milbrey y Patrick Shields. "Involving Low Income Parents in the Schools: A Role for Policy?” Phi Delta Kappan. 69.2 (2007): 156-60. 227. http://www.michigan.gov/documents/mde/PE_Toolkit_Spanish_Translation_37481 4.7.pdf

Sheldon, Steven. "Parents' Social Networks and Beliefs as Predictors of Parent Involvement". The Elementary School Journal. 102.4. (2002): 301-316. 
Valdés Cuervo, Ángel Alberto y Pedro Antonio Sánchez Escobedo. "Participación de los padres de alumnos de educación primaria en las actividades académicas de sus hijos”. Revista Electrónica de Investigación Educativa. 11.1. (2009): 3-18

Weiss, Heather; Suzanne Bouffard, Beatrice Bridglall y Edmund Gordon "Hacia un Nuevo enfoque sobre el involucramiento de la familia en la educación: Apoyar a las familias para promover la equidad en la educación”. Campaña por la Equidad Educativa, del Instituto Docente de la Universidad de Columbia. Diciembre de 2009. http:/ /www.hfrp.org/family-involvement/publications-resources 